\title{
FORMALDEÍDO GASOSO: A QUALIDADE DO AR EM BIBLIOTECAS E ESCOLAS
}

Ana Paula de Souza Figueiredo"; Rafael Rabello Cabral ${ }^{2}$; Júlia Faria da Rocha Pinheiro $^{2}$; Henrique Jordem Venial ${ }^{3}$; Murilo de Oliveira Souza ${ }^{4}$

${ }^{1}$ Graduanda em Licenciatura em Química no Instituto Federal de Educação, Ciência e Tecnologia Fluminense (IFF), Itaperuna-RJ.

${ }^{2}$ Técnicos em Química pelo Instituto Federal de Educação, Ciência e Tecnologia Fluminense (IFF), Itaperuna-RJ.

${ }^{3}$ Técnico em Anatomia no Centro de Ciências Agrárias e Engenharias, Universidade Federal do Espírito Santo (UFES), Alegre-ES.

${ }^{4}$ Professor de Química Analítica no Instituto Federal de Educação, Ciência e Tecnologia Fluminense (IFF), Itaperuna-RJ.

Email:m.quimic@gmail.com e murilo.souza@iff.edu.br

Recebido em: 15/11/2021 - Aprovado em: 15/12/2021 - Publicado em: 30/12/2021

DOI: 10.18677/EnciBio_2021D27

trabalho licenciado sob licença Creative Commons Attribution-NonCommercial-NoDerivatives 4.0 International License

\section{RESUMO}

A qualidade do ar tornou-se um assunto de grande atenção nas últimas décadas, no intuito de descobrir as principais fontes de poluentes atmosféricos de ambientes internos e externos, além de avaliar como estes compostos afetam os seres humanos. Apesar da ênfase dada aos poluentes externos, devido às doenças respiratórias, aos efeitos carcinogênicos e às mortes causadas pela exposição aos mesmos, neste trabalho foi dado destaque aos ambientes internos (principalmente escolas e bibliotecas), locais onde diversas pessoas passam grande parte do tempo diariamente. Dentre os poluentes contidos no ar, o formaldeído $\left(\mathrm{CH}_{2} \mathrm{O}\right)$ é um dos compostos mais abundantes nos ambientes internos e responsáveis por diversas doenças. Portanto, este trabalho teve como objetivo revisar o formaldeído no ar interno de escolas e bibliotecas, os efeitos e impactos do formaldeído na saúde humana, as metodologias de amostragem e coleta e, por fim, os instrumentos mais utilizados para a determinação deste composto. Ao final, concluiu-se que a emissão do formaldeído presente nas construções e mobília dos ambientes internos, gera consequências negativas à saúde. Verificou-se também que algumas doenças podem ser desencadeadas pelo longo período de exposição ao formaldeído. Desse modo, constatou-se que são necessários alguns cuidados para minimizar a emissão e exposição do formaldeído no ar de ambientes internos.

PALAVRAS-CHAVE: Ambientes Internos; Amostragem; Formaldeído; Qualidade do Ar; Saúde.

\section{GASEOUS FORMALDEHYDE: AIR QUALITY IN LIBRARIES AND SCHOOLS}

\begin{abstract}
Air quality has become a subject of great attention in recent decades, to discover the main sources of air pollutants from indoor and outdoor environments, in addition to assessing how these compounds affect humans. Despite the emphasis given to external pollutants, due to respiratory diseases, carcinogenic effects, and deaths caused by exposure to them, in this work, emphasis was placed on indoor environments (mainly schools and libraries), places where several people spend a


long period during the week. Among the pollutants contained in the air, formaldehyde $(\mathrm{CH} 2 \mathrm{O})$ is one of the most abundant compounds in indoor environments and is responsible for several diseases. Therefore, this work aimed to review formaldehyde in the air of schools and libraries, the effects and impacts of formaldehyde on human health, the methodologies of sampling and collection, and, finally, the most used instruments for the determination of this compound. In the end, it was concluded that the emission of formaldehyde present in buildings and furniture in indoor environments causes negative health effects. It was also found that some diseases can be triggered by a long period of exposure to formaldehyde. Thus, it was found that some precautions are necessary to minimize the emission and exposure of formaldehyde in indoor air.

KEYWORDS: Indoor air; Sample; Formaldehyde; Air Quality; Health.

\section{INTRODUÇÃO}

A química atmosférica tem se tornado um ramo da ciência de cunho interdisciplinar composta por estudiosos de diversas áreas como da química, da biologia, da física, da medicina e das ciências sociais, visto que os impactos ambientais estão interligados ao comprometimento socioambiental das empresas, dos órgãos públicos e da população. Desta forma, a qualidade do ar tornou-se alvo de grande atenção nas últimas décadas no intuito de identificar as fontes dos poluentes atmosféricos, assimilar como estes poluentes respondem à emissão e analisar como influenciam nos seres humanos, nos ecossistemas e no clima (ALVES; ACIOLE, 2012; SOUZA et al., 2021).

Quando se fala em poluição do ar, faz-se uma associação apenas aos poluentes presentes nas ruas, provenientes principalmente de automóveis e indústrias (poluição externa - outdoor). Esses poluentes continuam sendo emitidos por estas fontes e são prejudiciais à saúde humana, bem como para os monumentos, edifícios e ao planeta. Entretanto, a partir da década de 60, iniciou-se uma grande preocupação com a qualidade do ar interno (poluição interna - indoor) (SOFUOGLU et al., 2011; SÁNCHEZ et al., 2012; WOLKOFF, 2013; SCHIEWECK et al., 2018; LI et al., 2019; NUNES et al., 2019; SÁNCHEZ et al., 2020; SOUZA et al., 2020; 2021).

O interesse pelo estudo da qualidade do ar em ambientes internos (indoor), surgiu após tomar-se conhecimento sobre o crescimento de microrganismos mesmo em ambientes livres da "sujeira da rua". A qualidade do ar não está relacionada somente com a poluição outdoor, a concentração de aldeídos (dentre estes, o formaldeído - $\mathrm{CH}_{2} \mathrm{O}$ ) em ambientes indoor pode ser 13 vezes maior que nos ambientes outdoor, algo extremamente preocupante visto que as pessoas passam $90 \%$ do tempo em ambientes fechados (casas, escolas, trabalhos, entre outros) e 7 $\%$ no interior de veículos (KLEPEIS et al., 2001). Além disso, a otimização de espaços, o aumento da quantidade de pessoas por metro quadrado, a baixa ventilação, associados à quantidade de materiais emissores de poluentes dentro desses ambientes, contribuem cada vez mais para o aumento e acúmulo de diversos compostos nocivos em atmosferas internas (SOUZA et al., 2021).

Sabe-se que o formaldeído é considerado um dos principais compostos responsáveis por diversas doenças (por exemplo, câncer nasofaríngeo e problemas respiratórios) e que está presente em diversos lugares como, por exemplo, em escolas e bibliotecas (SOUZA et al., 2021). Desta forma, a avaliação do formaldeído no ar de escolas e bibliotecas faz-se importante pelo fato de serem locais utilizados diariamente por estudantes, professores e demais pessoas da comunidade 
acadêmica. Essas pessoas permanecem por horas nesses locais sendo, assim, sujeitas a um maior tempo de exposição ao formaldeído.

Portanto, o objetivo deste trabalho foi avaliar as fontes de emissão e os efeitos nocivos da exposição ao formaldeído em espaços internos como escolas e bibliotecas. Além disso, os principais métodos de amostragem e análise de formaldeído gasoso em espaços internos foram discutidos. Por fim, neste artigo é apresentado um panorama geral das principais legislações e diretrizes (nacionais e internacionais) que estabelecem os limites de exposição ao formaldeído em ambientes internos.

\section{FONTES DE EMISSÃO E REMEDIAÇÃO DE FORMALDEÍDO DO AR}

Segundo a Ficha de Informação Toxicológica divulgada pela Companhia Ambiental do Estado de São Paulo (CETESB, 2017), o formaldeído, também conhecido como metanal ou aldeído fórmico, é um gás incolor, inflamável e altamente reativo em condições normais de temperatura e pressão. No ar ambiente, o formaldeído se polimeriza rapidamente formando um precipitado branco denominado de paraformaldeído e, devido às suas características físicas e seu baixo custo, compreende-se entre as 25 substâncias químicas que são produzidas com maior abundância no mundo. Somado a isso, o formaldeído pode ser emitido por diversas fontes naturais (incêndios e queima de madeira), por usinas de energia, fábricas, incineradores e por veículos não equipados com catalisadores que auxiliam na oxidação dos gases de escape (dentre estes, o formaldeído) a compostos inócuos ( $\mathrm{CO}_{2}, \mathrm{H}_{2} \mathrm{O}$ e $\mathrm{N}_{2}$ ). Destaca-se ainda, que o formaldeído é um dos compostos voláteis que possui formação nos primeiros estágios de decomposição de resíduos vegetais no solo, e ocorre naturalmente em frutas e outros alimentos. As concentrações de formaldeído no ar de espaços internos são geralmente menores que $0,02 \mathrm{mg} \mathrm{m}^{-3}$ (em áreas urbanas). Entretanto, estes valores podem atingir concentrações acima de $0,1 \mathrm{mg} \mathrm{m}^{-3} \mathrm{em}$ ambientes urbanizados e com fluxo intenso de veículos, ou durante episódios graves de inversão térmica (CETESB, 2017).

O formaldeído em bibliotecas e escolas vem tomando destaque nas últimas décadas devido às diversas fontes de emissão deste composto como, por exemplo, papéis e tintas usadas para confeccionar os livros, mobília de madeira e materiais usados na construção e reforma destes ambientes (TRINKLEY, 2001; MARCHAND et al., 2006). A Figura 1 apresenta a emissão de formaldeído de papéis e compensados de madeira (estantes) presentes em uma biblioteca.

FIGURA 1. Ilustração de um ambiente indoor (biblioteca) demonstrando a emissão de formaldeído gasoso.

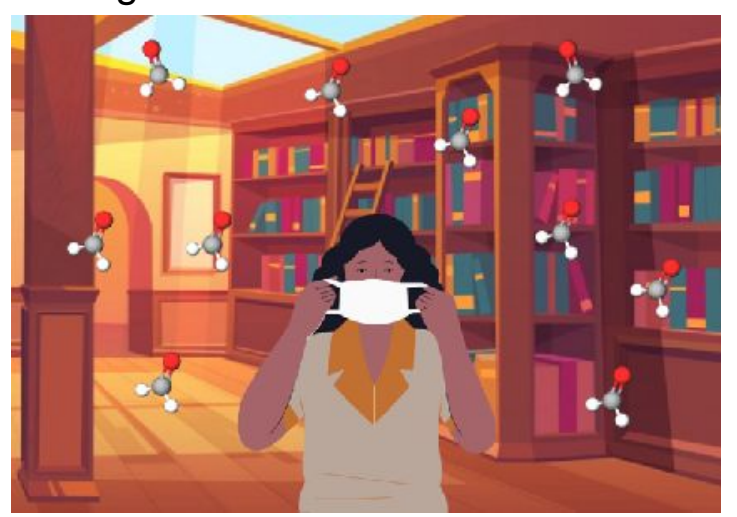

Fonte: Autores (2021). 
A espuma para isolamento de ureia-formaldeído e produtos de madeira (particularmente os compensados) são as fontes emissoras mais influentes de formaldeído (MARCHAND et al., 2006; KABIR; KIM, 2011; DIODIU et al., 2016; SÁNCHEZ et al., 2020). Além disso, em alguns compensados de madeira se utiliza uma resina fenólica que provavelmente se degrada ao longo do tempo e libera formaldeído. Há indícios na literatura que sugerem que a madeira colada com resinas fenólicas emite cerca de 10 vezes menos formaldeído do que aquela colocada com uma resina de uréia-formaldeído (TRINKLEY, 2001). Sabe-se também que o formaldeído é frequentemente adicionado a diversos produtos, como papel de parede, cosméticos, detergentes, conservantes e fungicidas, sendo estes produtos possíveis emissores de formaldeído (SOUZA et al., 2021).

As escolas e bibliotecas são ambientes propícios à emissão de formaldeído devido à presença de papéis, livros, móveis de madeira, estofados, entre outros. Associado a isso, esses lugares frequentemente não possuem um sistema de ventilação adequado, expondo toda a comunidade acadêmica a diferentes compostos orgânicos voláteis (em especial, o formaldeído). Em escolas primárias, onde o ensino é integral, as crianças ficam expostas por longos períodos e, por serem mais sensíveis, o risco é maior já que possuem o sistema imunológico mais ativo e respiram mais ar do que os adultos (ALVES; ACIOLE, 2012).

Segundo Alves e Aciole (2012), foi avaliada a qualidade do ar de 71 salas de aula em 17 jardins de infância coreanos. As concentrações médias de formaldeído no interior das escolas de ensino pré-escolar que ficam localizadas em áreas urbanas, foram de 44,6 $\pm 40,9 \mathrm{~g} \mathrm{~m}^{-3}$, enquanto na parte externa, as concentrações foram de 26,4 $\pm 36,1 \mathrm{~g} \mathrm{~m}^{-3}$. Já em localidades rurais, as concentrações médias no interior dos jardins de infância foram de 39,4 $\pm 25,7 \mathrm{~g} \mathrm{~m}^{-3}$ e no exterior foram de 7,6 $\pm 2,7 \mathrm{~g} \mathrm{~m}^{-3}$. A diferença entre as concentrações do interior e do exterior foram elevadas, sugerindo assim a existência de inúmeras fontes de emissão interna.

Vários estudos têm buscado formas de remover o formaldeído de ambientes confinados. Sistemas de filtração baseados em adsorção e fotocatálise heterogênea têm apresentado resultados promissores para a remoção de formaldeído de espaços internos. Além disso, a pandemia do coronavírus iniciada em 2019, alertou para os perigos dos ambientes fechados, os quais podem concentrar fungos, bactérias e vírus; além de compostos nocivos, como é o caso do formaldeído. Neste sentido, algumas escolas e estabelecimentos públicos têm usado a medida de $\mathrm{CO}_{2}$ para avaliar o grau de ventilação destes ambientes. Porém, pouco ainda se fala sobre o monitoramento de poluentes químicos como, por exemplo, o formaldeído para a avaliação da qualidade do ar de bibliotecas e escolas (ALVES; ACIOLE, 2012; SOUZA et al., 2021).

Além do mais, a redução da poluição do ar em ambientes internos e a remoção de formaldeído pode ser realizada por um processo denominado fitorremediação. Ferreira e Cardoso (2013) utilizaram algumas plantas naturais ou modificadas biotecnologicamente para ajudar na remediação de um ecossistema poluído. Os resultados demonstraram que as relações simbióticas entre plantas e microrganismos apresentam respostas eficientes ao metabolizar substâncias nocivas em compostos inócuos. Além da alta eficácia e baixo custo, o procedimento pode ser realizado em diversos tipos de espaços internos como, por exemplo, em escolas e bibliotecas.

\section{LIMITES DE EXPOSIÇÃO AO FORMALDEÍDO EM AMBIENTES INTERNOS}

O Instituto Nacional de Segurança e Saúde Ocupacional, segundo Veronez et ENCICLOPÉDIA BIOSFERA, Centro Científico Conhecer - Jandaia-GO, v. 18 n.38; p. 362 
al. (2010), passou a considerar, desde 1993, o formaldeído como fator de risco carcinogênico aos trabalhadores que convivem com essa substância em todas as suas atividades diárias. Com isso, foram estabelecidos novos padrões para a tolerância dessa substância $(0,016$ ppmv segundo $\mathrm{NIOSH})$, que adequam o seu nível de concentração ao tempo presente no ambiente de trabalho (Tabela 1).

No Brasil, a Norma Técnica 002, Resolução 09 da Agência Nacional de Vigilância Sanitária (ANVISA, 2003), determina os padrões referenciais de qualidade do ar interno em ambientes climatizados artificialmente de uso público ou privado. Contudo, a norma não estabelece os limites máximos para exposição ao formaldeído, há somente a orientação quanto à utilização de produtos de limpeza, materiais de construção, acabamentos e mobiliários que emitam uma quantidade menor de formaldeído. No entanto, a Norma Regulamentadora 15 do Ministério do Trabalho e Emprego (NR-15 / Anexo XI - Atividades e Operações Insalubres) estabelece limites de exposição ocupacional ao formaldeído a que os trabalhadores podem estar expostos no ambiente de trabalho. A Tabela 1 apresenta os limites estabelecidos por algumas legislações de órgãos nacionais e internacionais (Figura 2) para a exposição de formaldeído em ambientes internos.

TABELA 1. Limites de exposição ocupacional para formaldeído regulados em ambientes internos: ppmv.

\begin{tabular}{|c|c|c|c|}
\hline 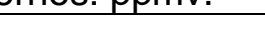 & \multicolumn{3}{|c|}{ Formaldeído } \\
\hline & TM & CP & LMT \\
\hline OMS & $0,08^{\mathrm{a}}(0,10)^{\mathrm{b}}(100)^{\mathrm{c}}$ & $0,08^{a}(0,10)^{b}(100)^{c, d}$ & -- \\
\hline ACGIH (EUA) & $0,10^{a}(0,12)^{b}(120)^{c}$ & - & $\underset{c}{0,30^{a}(0,37)^{b}(370)}$ \\
\hline OSHA (EUA) & $0,75^{\mathrm{a}}(0,92)^{\mathrm{b}}{ }_{(920)}^{\mathrm{c}}$ & $2,00^{a}(0,00246)^{b}(2,46)^{c, e}$ & -- \\
\hline $\mathrm{NIOSH}$ (EUA) & $0,016^{\mathrm{a}}(0,02)^{\mathrm{b}}(20)^{\mathrm{c}}$ & - & $\underset{c}{0,10^{a}}{ }_{(0,12)}^{b}(120)$ \\
\hline DFG (Alemanha) & $0,30^{a}(0,37)^{b}(370)^{c}$ & - & -- \\
\hline JSOH (Japão) & $0,10^{a}(0,12)^{b}(120)^{c}$ & - & -- \\
\hline MOEL (Coréia) & $0,50^{\mathrm{a}}(0,61)^{\mathrm{b}}(610)^{\mathrm{c}}$ & - & -- \\
\hline APA (Portugal) & $0,08^{a}(0,0001)^{b}(0,10)^{c}$ & - & -- \\
\hline NR 15 (Brasil) & $1,60^{a}(0,0023)^{b}(2,30)^{c}$ & - & -- \\
\hline
\end{tabular}

Valores em: ${ }^{2} \mathrm{ppmv},{ }^{b} \mathrm{mg} \mathrm{m}^{-3} \mathrm{e}^{\mathrm{c}} \mu \mathrm{g} \mathrm{m}^{-3}$

TM: Tempo médio de 40 horas semanais de trabalho.

CP: Limite de exposição a curto prazo ${ }^{\mathrm{d}} 15 \mathrm{~min}^{\mathrm{e}} 30 \mathrm{~min}$.

LMT: Limites máximos de tolerância que não devem ser excedidos.

OMS (WHO) - Organização Mundial de Saúde, 1996.

ACGIH - Conferência Americana de Higienistas Industriais Governamentais, 1971.

OSHA - Administração de Segurança e Saúde Ocupacional, 1990.

NIOSH - Instituto Nacional de Segurança e Saúde Ocupacional, 1994.

DFG - Fundação Alemã de Pesquisa, 2000.

JSOH - Sociedade Japonesa de Saúde Ocupacional, 1988.

MOEL - Ministério do Emprego e Trabalho, 2010.

APA - Agência Portuguesa do Ambiente, 2010.

NR 15 - Ministério da Saúde Brasil, 2019. 
FIGURA 2. Órgãos nacionais e internacionais que regulamentam limites de exposição de formaldeído em ambientes internos.

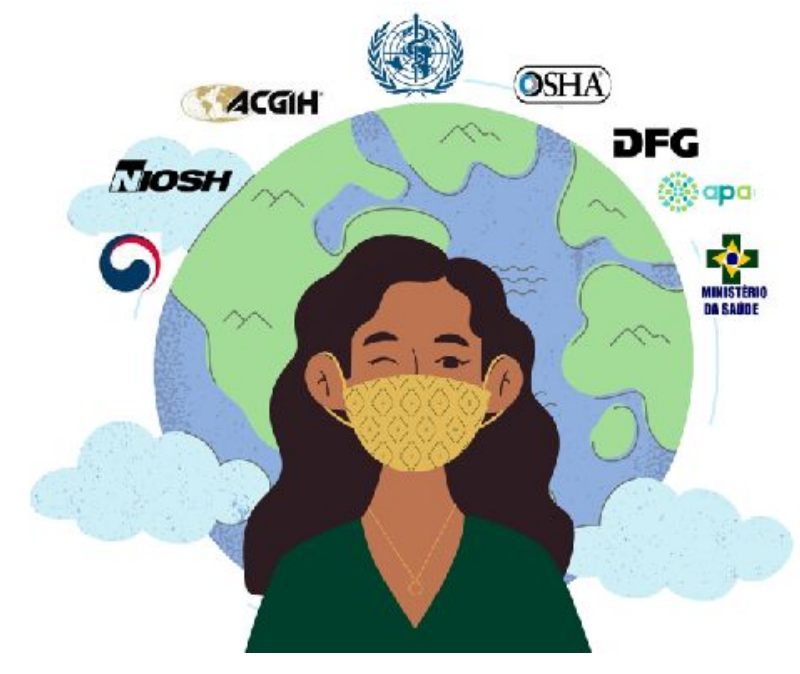

Fonte: Autores (2021).

Os Limites de Exposição Ocupacional da norma reguladora (NR 15) são muito mais flexíveis do que aqueles definidos por competências internacionais como OSHA e DFG. Ainda que estes limites não sejam utilizados como evidências relativas de toxicidade e estimativas de quantitativas de ameaça à saúde, estes são valores que validam níveis de segurança à saúde, para adoção de sistemas de proteção e procedimentos de manuseio pertinentes (TSAl, 2019). Entretanto, esta norma não está direcionada a escolas, bibliotecas, casas, entre outros ambientes internos; estando relacionada somente a ambientes ocupacionais de cunho industrial.

Ferreira e Cardoso (2013) determinaram a qualidade do ar em 51 escolas de educação básica (tanto no interior como no exterior das salas de aula) na cidade de Coimbra em Portugal, durante as quatro estações do ano, entre 2010 e 2011. Os resultados obtidos mostraram que diversos poluentes se encontravam em concentrações acima do limite permitido pela legislação portuguesa, porém, não foram obtidos níveis relevantes de formaldeído que fossem prejudiciais aos estudantes.

Em suma, a qualidade do ar de espaços internos deve ser foco de grande preocupação pelos órgãos de fiscalização, com o propósito de incentivar medidas que certifiquem a qualidade do mesmo. Além disso, deve-se garantir uma boa ventilação destes ambientes para que haja dispersão destes poluentes. Desta forma, é necessário reformular as normas e legislações vigentes, além de promover a criação de políticas públicas eficazes, que assegurem a redução dos impactos ocasionados por contaminação química em ambientes internos (OLMO et al., 2011).

\section{RISCOS À SAÚDE DEVIDO A EXPOSIÇÃO AO FORMALDEÍDO}

O formaldeído está presente em baixas concentrações em grande parte dos seres vivos (quantidades fisiológicas de formaldeído se formam no organismo a partir de reações químicas), porém, a exposição a elevadas concentrações traz efeitos negativos à saúde. Vários estudos foram feitos após a década de 90 , associando altas concentrações de formaldeído à problemas respiratórios em crianças, demonstrando o risco deste composto em escolas e bibliotecas. Estes 
estudos apontaram que o formaldeído não é um agente desencadeador da asma infantil, mas pode provocar inflamações nas mucosas das vias respiratórias, produzindo citocinas da categoria das interleucinas que podem desencadear crises asmáticas (ALVES; ACIOLE, 2012).

Como relatado nas seções anteriores deste trabalho, o formaldeído é frequentemente adicionado a diversos produtos, como papel de parede, cosméticos, detergentes, conservantes e fungicidas. Devido a seu baixo ponto de ebulição ($19,5^{\circ} \mathrm{C}$ ), quando a temperatura ambiente aumenta, o formaldeído contido nesses produtos é volatilizado, contaminando o ar interior (SAKAMOTO et al., 1999). Desta forma, devido a alta volatilidade do formaldeído, maior quantidade é inalada por aqueles que manuseiam produtos contendo o mesmo. Além disso, o formaldeído em altas concentrações, pode causar fortes dores de cabeça, irritação dos olhos, tosse, falta de ar, vertigem, dificuldade para respirar, edema pulmonar, laringite, bronquite e pneumonia (MENDELL et al., 2007).

A contaminação de espaços internos com o formaldeído é uma ameaça à saúde humana. A forte reatividade desta molécula reside no carbono eletrolítico (com densidade de carga positiva) presente no grupo carbonila, que pode ser atacado rapidamente por grupos tiol e amino com alta densidade eletrônica (Figura 3). A reação de formaldeído com esses grupos nucleofílicos presentes nos ácidos nucléicos e nas proteínas pode causar várias lesões ao organismo, conduzindo a várias doenças (anemia de Fanconi, carcinogênese hepática, falência renal e síndrome de Ruijs-Aalfs) (REINGRUBER; PONTEL, 2018).

FIGURA 3. Reação de formaldeído com grupos tiol e amino.
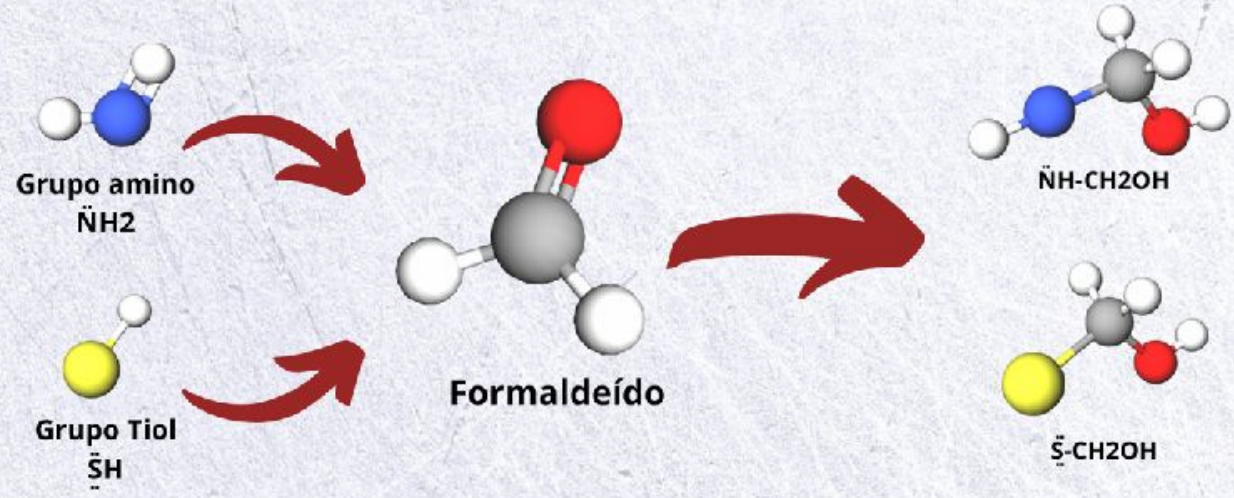

Fonte: Autores (2021).

Além da contaminação através das fontes internas, o formaldeído compreendido no ar externo pode ser arrastado pela ação dos ventos e/ou por outros agentes, confinando-o em espaços internos. A poluição industrial, combustão de biomassa, fumaça de carro, entre outros podem contribuir significativamente para a poluição interna com formaldeído. Neste sentido, a Organização Mundial da Saúde (OMS) estabeleceu uma diretriz que limita a $0,08 \mathrm{ppm}_{\mathrm{v}}$ de formaldeído para uma exposição de 30 minutos (Tabela 1) (OMS, 1996). 
Vários trabalhos já correlacionaram o formaldeído com afecções respiratórias e cutânea, como: a asma; sintomas respiratórios crônicos e alergias; deterioração leve, porém significativa, da função pulmonar; dermatite de contato; entre outros (SAKAMOTO et al., 1999; WOLKOFF, 2013; BASSIG et al., 2016; REINGRUBER; PONTEL, 2018; YON et al., 2019; ZHANG et al., 2019). De acordo com Sakamoto et al. (1999) o limiar de odor (consciência olfativa) para formaldeído varia entre 0,05 e 1 ppmv. Já o grau de irritação é diretamente proporcional à concentração e duração da exposição ao formaldeído (irritação ocular, 0,01 - 2,0 ppmv; irritação nas vias aéreas superiores, $0,1-25$ ppmv). Os efeitos pulmonares (como tosse, aperto no peito e dispnéia) e nas vias aéreas inferiores (traqueia, brônquios, bronquíolos e alvéolos) foram observados em concentrações entre 5 e 30 ppmv de formaldeído.

Veronez et al. (2010), apontaram que o Conselho Nacional de Pesquisa (NRC) dos EUA, em 1988, determinou que o formaldeído na concentração de 0,1 a 2,0 ppmv $\left(9,15\right.$ a $\left.3,0 \mathrm{mg} \mathrm{m}^{-3}\right)$ é irritante à mucosa ocular, provoca irritação às mucosas do nariz, cavidade nasal, faringe e laringe em concentração de 0,1 ppmv, trazendo consequências mais graves na traqueia, brônquios e pulmões em concentrações entre 5 a 30 ppmv $\left(7,5\right.$ a $\left.45 \mathrm{mg} \mathrm{m}^{-3}\right)$.

O formaldeído afeta principalmente, os olhos e as vias aéreas superiores (cavidade nasal, cavidade oral, faringe e laringe). As irritações oculares e da via aérea superior são as queixas mais comuns de pessoas que vivem ou trabalham em ambientes onde materiais contendo compostos de formaldeído são usados em grande quantidade (SAKAMOTO et al., 1999).

O formaldeído, em 2004, foi categorizado no grupo 1 (carcinogênico para humanos, segundo as evidências epidemiológicas do desenvolvimento de câncer nasofaríngeo) pela Agência Internacional de Pesquisa em Câncer (COGLIANO et al., 2005). Além disso, foi categorizado com probabilidades cancerígenas à humanos (1B) pela Agência de Proteção Ambiental (USEPA, 1999), baseado em suficientes evidências em animais.

\section{AMOSTRAGEM DE FORMALDEÍDO GASOSO}

A amostragem do formaldeído gasoso pode ser realizada de forma ativa ou passiva. A amostragem ativa é realizada com uma bomba de sucção conectada a um tubo de vidro, aço inoxidável ou outro material, permitindo que um determinado volume de ar passe pelo material adsorvente (SCHIRMER et al., 2011). Devido à alta sensibilidade (partes por bilhão, $\mathrm{ppb}_{\mathrm{v}}$ e trilhões, $\mathrm{ppt}_{\mathrm{v}}$ ) e velocidade de amostragem, a amostragem ativa é preferida para análise ambiental (DIODIU et al., 2016; MOKALLED et al., 2019; NUNES et al., 2019; SOUZA et al., 2020; SÁNCHEZ et al., 2020).

A amostragem passiva ocorre com a difusão do ar no tubo adsorvente não sendo necessário o uso de bomba de sucção para forçar a passagem do ar pelo amostrador (ou seja, não envolve o movimento ativo do ar para dentro do amostrador) (SOUSA et al., 2015). Os amostradores de fluxo passivo são empregados para medir o formaldeído no ar de maneira simples, eficiente e de baixo custo (apresentando uma fácil construção e operação). A limitação destaque quanto à amostragem passiva é o longo tempo de amostragem e a viabilidade de contaminação com outras substâncias (devido ao alto tempo em exposição) (SALTHAMMER et al., 2010; SOUSA et al., 2015).

\section{ANÁLISE DE FORMALDEÍDO GASOSO}

A amostragem, o preparo de amostra (pré-tratamento) e a análise de 
formaldeído na atmosfera não são triviais. A baixa concentração de formaldeído no ambiente requer a necessidade de uma pré-concentração e, para isto, é fundamental realizar a amostragem de um grande e conhecido volume de gás. No caso do formaldeído, a análise pode ser realizada de forma direta (sem exigir prétratamento da amostra) ou indireta (requerem uma ou mais etapas de pré-tratamento da amostra antes da determinação) (Figura 4).

FIGURA 4. Análise de formaldeído gasoso.

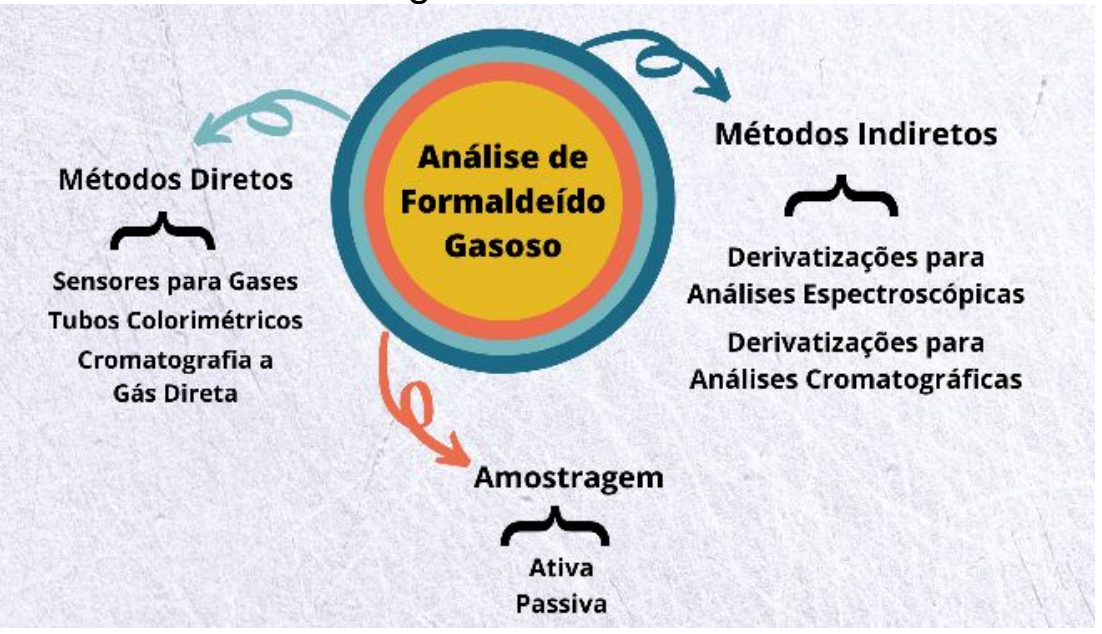

Fonte: Autores (2021)

\section{Análise direta de Formaldeído}

A análise direta (também chamada de análise em tempo real ou online) do formaldeído reduz o risco de contaminação durante a determinação, considerando que os erros mais abundantes durante uma análise estão associados à etapa de amostragem e pré-tratamento das amostras. Nesta revisão foram considerados como métodos diretos aqueles em que o formaldeído é analisado sem qualquer etapa de pré-concentração e/ou derivatização ou que passaram por um prétratamento simples como, por exemplo, uma coleta em bolsa de plástico ou uma rápida reação de derivatização para análise subsequente.

\section{Sensores para gases}

Os sensores para gases são instrumentos que possibilitam a determinação direta de inúmeros compostos gasosos, dentre estes o formaldeído no ar. As moléculas do gás interagem com a superfície do sensor, gerando informação química medida por um transdutor (ESCALONA et al., 2012). Os sensores expressam benefícios quanto a portabilidade, facilidade de automação, possibilidade de miniaturização e baixo custo. Contudo, os sensores podem apresentar limitações como, por exemplo, a falta de seletividade e baixa detectabilidade (LOWINSOHN; BERTOTTI, 2006).

Entre os sensores para gases, alguns são monitores comerciais simples e portáteis (eletroquímicos e fotométricos) empregados para a determinação direta de formaldeído no ar (MIKSCH et al., 1981; ALVES; ACIOLE, 2012). Estes equipamentos são manuseados com fins de monitoramento de formaldeído em indústrias e em ambientes internos. Os monitores fotométricos são dispositivos pequenos onde sucede a reação química entre um reagente específico e o formaldeído, obtendo como resultado um derivado colorido. A intensidade da cor é diretamente proporcional à concentração de formaldeído no ar, sendo apresentado em um leitor contido no monitor portátil (ALVES; ACIOLE, 2012; HLADOVÁ et al., 
2019). Pode-se destacar o método IP-6B da EPA (empregando o reagente pararosanilina), que é utilizado em diversos analisadores contínuos comerciais para aferir a concentração de formaldeído no ar (GODISH, 2010).

\section{Tubos colorimétricos}

O tubo colorimétrico ou tubo detector é fechado e aberto somente no momento da medição, para que sejam mínimas as possibilidades de contaminação. Este método é realizado através de uma bomba manual pequena e leve feita de polipropileno, para aspirar o ar para dentro do tubo. Quando o ar atravessa o tubo, ocorre uma derivatização, onde uma substância química e o formaldeído reagem quimicamente no interior do tubo, o que acaba por alterar a coloração do formaldeído. A leitura da concentração de formaldeído é realizada propriamente no tubo, por meio do comprimento de onda da cor obtida (FENG et al., 2005).

Para determinação de formaldeído em ambientes internos, os tubos colorimétricos podem apresentar inadequação, este fator dar-se-á em função da baixa precisão (RSD $= \pm 25 \%$ ), baixa sensibilidade, baixa detectabilidade, alto tempo necessário para os procedimentos de estabilização/reação, padronização e da limpeza do instrumento (OBERMEIER et al., 2015).

\section{Cromatografia a gás direta}

A análise direta de formaldeído pode ser efetuada empregando a cromatografia a gás (gas chromatography - GC) com os detectores por ionização em chama (flame ionization detector - FID), por condutividade térmica (thermal conductivity detectors - TCD) e por fotoionização. Quando estes detectores estão associados a uma coluna cromatográfica adequada, torna-se possível separar e analisar o formaldeído no ar, apresentando uma abordagem analítica de alta adaptabilidade (SOUZA et al., 2021).

$\mathrm{O}$ alto custo destes equipamentos e a baixa sensibilidade para injeções diretas se tornam um desafio na análise do formaldeído no ar. Além disso, devido à presença de somente uma ligação $\mathrm{CH}$, o formaldeído possui baixo sinal analítico, o que reduz a sensibilidade ao usar o detector FID (LAGHRIB et al., 2019). Ademais, alguns estudos indicam a possível reatividade do $\mathrm{O}_{3}$ presente no ar com os diferentes tipos de coluna do cromatógrafo a gás (PASETTO et al., 2019).

\section{Análise indireta de Formaldeído}

Considerando as restrições relatadas no decorrer da análise direta de formaldeído no ar, grande parte dos trabalhos encontrados na literatura demandam uma ou mais etapas de pré-tratamento antes da determinação, não havendo o fornecimento imediato dos resultados (métodos indiretos). A derivatização (com DNPH, ácido cromotrópico, entre outros) e pré-concentração (utilizando adsorventes, como a sílica gel ou C18 [substrato de sílica ligado ao octadecilsilano]) do formaldeído são as etapas de preparo de amostra mais utilizadas (ROTHWEILER et al., 1991; PERRAUD et al., 2016; MAJCHRZAK et al., 2018; SOUZA et al., 2021).

\section{Derivatizações para análises espectroscópicas}

Os métodos de derivatização colorimétrica constituem em uma reação química entre um agente derivatizante e o formaldeído dispondo-se de um novo composto, que pode ser analisado por espectroscopia de absorção molecular UVVIS ou da espectrofluorimetria. A análise do formaldeído sem a derivatização é muito complexa devido à ausência de grupos cromóforos ou fluoróforos: substâncias 
que interagem com a luz, ou seja, que são encarregados da interação com a radiação eletromagnética (DUGHERI et al., 2018).

A derivatização ocorre quando há a interação do formaldeído com um derivatizante específico, que pode estar impregnado em cartuchos, filtros ou dissolvidos em uma solução líquida (impinger), de forma passiva ou ativa (SALTHAMMER et al., 2010; SOUSA et al., 2015). Alguns reagentes derivatizantes já foram empregados para a derivatização de formaldeído, a saber: pararosanilina (HLADOVÁ et al., 2019), ácido 7-amino-5-hidroxi-2-naftaleno-sulfâmico (J-Acid) (ARAGON et al., 2000), p-fenilenodiamina (HALKET; ZAIKIN, 2005), entre outros (VOGEL et al., 2000; FU et al., 2019;). Os principais reagentes derivatizantes aplicados para a determinação de formaldeído do ar de ambientes interiores estão listados na Tabela 2.

TABELA 2. Principais reagentes derivatizantes utilizados na determinação de formaldeído de forma indireta no ar de ambientes internos.

\begin{tabular}{|c|c|c|c|}
\hline Amostragem & Derivatizante & Detector/Técnica Analítica & $\begin{array}{c}\text { Comprimento de } \\
\text { Onda }\end{array}$ \\
\hline \multirow{3}{*}{$\begin{array}{l}\text { Ativa } \\
\text { ou } \\
\text { Passi } \\
\text { va }\end{array}$} & $\begin{array}{l}\text { Ácido cromotrópico } \\
\quad \text { (1,8-di- } \\
\text { hidroxinaftaleno-3,6- } \\
\text { ácido- dissulfônico) }\end{array}$ & $\begin{array}{c}\text { Detector UV- } \\
\text { Vis/Espectroscopia de } \\
\text { absorção molecular UV-VIS }\end{array}$ & $580 \mathrm{~nm}$ \\
\hline & $\begin{array}{l}\text { Reagente de Nash } \\
\text { (acetilacetona, } \\
\text { acetato de amônio } \\
\text { e ácido acético) }\end{array}$ & $\begin{array}{c}\text { Detector UV- } \\
\text { Vis/Espectroscopia de } \\
\text { absorção molecular UV-VIS } \\
\text { ou espectrofluorimetria }\end{array}$ & 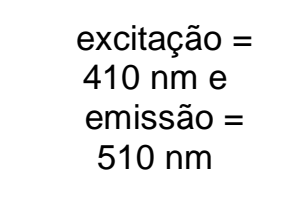 \\
\hline & $\begin{array}{l}\text { Purpald (4-amino- } \\
\text { 3- hidrazino-5- } \\
\text { mercapto-4H- } \\
\text { 1,2,4-triazol) }\end{array}$ & $\begin{array}{c}\text { Detector UV- } \\
\text { Vis/Espectroscopia de } \\
\text { absorção molecular UV-VIS }\end{array}$ & $\mathrm{nm}^{549}$ \\
\hline
\end{tabular}

Um método colorimétrico abrange uma singularidade muito grande, como, por exemplo, o reagente de Nash, específico para formaldeído e, com isso, não deve ser utilizado para aferir outros compostos. No entanto, existem reagentes que podem manifestar pouca especificidade (como, por exemplo, o DNPH), reagindo com vários aldeídos e cetonas. Essa distinção é delicada, uma vez que a carbonila $(C=0)$ é o centro reativo comum de todos compostos carbonílicos (DUGHERI et al., 2018). Ademais, os métodos colorimétricos fornecem uma baixa sensibilidade e estão suscetíveis a uma variedade de interferências que suprimem sua finalidade. Neste sentido, a derivatização relacionada aos métodos cromatográficos (sensíveis e com alta detectabilidade) tornou-se a abordagem analítica mais importante para separação e determinação de vários aldeídos (em destaque, o formaldeído) (SOUZA et al., 2020; 2021).

\section{Derivatizações para análises cromatográficas}

Atualmente, as técnicas cromatográficas a gás ou a líquido com diferentes detectores têm sido usadas para a separação e determinação de vários aldeídos (dentre eles, o formaldeído). Para conhecimento do autor foi publicado recentemente ENCICLOPÉDIA BIOSFERA, Centro Científico Conhecer - Jandaia-GO, v.18 n.38; p. 369 2021 
uma revisão que aborda as principais metodologias analíticas para análise de diferentes aldeídos e cetonas em ambientes internos (SOUZA et al., 2021). Entretanto, nesta seção do artigo será abordado somente a análise de formaldeído por cromatografia líquida de alta eficiência (HPLC) por ser a técnica analítica mais difundida.

Os principais critérios a serem considerados para utilização da técnica de cromatografia líquida de alta eficiência para a separação e análise de aldeídos (incluindo o formaldeído), são as características e propriedades do reagente derivatizante escolhido. O produto deve ser estável, de possível separação e detecção, e a reação com o formaldeído tem de ser rápida e quantitativa, com um derivatizante não muito volátil (VOGEL et al., 2000).

Os reagentes derivatizantes mais empregados para a análise de formaldeído no ar de espaços internos são as hidrazinas, sendo a 2,4-dinitrofenil-hidrazina (DNPH) a principal hidrazina empregada. Várias são as metodologias que utilizam o DNPH como agente derivatizante do formaldeído: EPA TO-11A, a NIOSH e o IP-6A. Além disso, há a possibilidade de impregnar o DNPH em diferentes adsorventes, sendo o C-18 e a sílica gel os mais usados para pré-concentração de compostos de baixo peso molecular, como o formaldeído (SOUZA et al., 2021).

Em uma amostragem de formaldeído no ar de ambientes indoor, os cartuchos amostradores são preenchidos com o adsorvente sílica gel e DNPH, e são acoplados à uma bomba que succiona o ar (ARAGON et al., 2000; SOUZA et al., 2020). Em seguida, o formaldeído é extraído utilizando um solvente adequado e injetado no HPLC, para separação e posterior análise do formaldeído derivatizado (SOUZA et al., 2020).

\section{CONSIDERAÇÕES FINAIS}

A emissão do formaldeído presente nas construções e mobílias dos ambientes internos gera consequências negativas à saúde, e a exposição a longo prazo ocasiona o surgimento de várias doenças. Sendo assim, os cuidados para minimizar a emissão e exposição de formaldeído no ar de bibliotecas e escolas devem ser feitos corretamente e com frequência, visto que diferentes públicos circulam nestes espaços, dentre eles, os estudantes mais novos que possuem um sistema imunológico mais ativo e respiram mais ar do que os adultos. Portanto, a implementação de sistemas de recirculação de ar mais eficientes e sistemas de tratamento e purificação de ar contaminado, são estratégias eficientes para a dispersão e/ou destruição do formaldeído no ar de bibliotecas e escolas. Além disso, os materiais e utensílios utilizados nas construções de escolas e bibliotecas deveriam ser repensados, a fim de reduzir ou eliminar as fontes emissoras de formaldeído destes ambientes. Por fim, políticas de incentivo ao monitoramento de formaldeído e outros compostos voláteis nesses ambientes confinados devem ser realizadas para o melhor entendimento dos efeitos nocivos de formaldeído e demais poluentes à saúde dos estudantes, professores e outros trabalhadores destes locais.

\section{REFERÊNCIAS}

ACGIH - American Conference of Governmental Industrial Hygienists. Documentation of the threshold limit values for substances in workroom air. Cincinnati: ACGIH, $1971 . \quad$ Disponível em: https://hero.epa.gov/hero/index.cfm/reference/details/reference_id/9810. Acesso em: 20 out. 2020. 
ALVES, C. A.; ACIOLE, S. D. G. Formaldeído em escolas: uma revisão. Química Nova, v. 35, n. 10, p. 2025-2039, 2012. Disponível em: https://doi.org/10.1590/S0100-40422012001000024. Acesso em: 05 nov. 2021. doi: 10.1590/S0100-40422012001000024.

ANVISA - Agência Nacional de Vigilância Sanitária. Legislação sobre a qualidade do ar interior. Brasil: Resolução RE/ANVISA no 9, de 16 de janeiro de 2003. Disponível em: https://www.saude.mg.gov.br/images/documentos/RES_RE_09.pdf. Acesso em: 05 nov. 2021.

APA - Agência Portuguesa do Ambiente. Qualidade do Ar em Espaços Interiores: Um Guia Técnico. Amadora: APA, 2010. Disponível em: https://webstorage.cienciaviva.pt/public/pt.cienciaviva.www/divulgacao/Manual_QAI_ APA_Maio2010.pdf. Acesso em: 10 fev. 2021.

ARAGON, P.; ATIENZA, J.; CLIMENT, M. D. Analysis of organic compounds in air: a review. Critical Reviews in Analytical Chemistry, v. 30, n. 2-3, p. 121-151, 2000. Disponível em: https://doi.org/10.1080/10408340091164207. Acesso em: 30 jan. 2021. doi: $10.1080 / 10408340091164207$.

BASSIG, B. A.; ZHANG, L.; VERMEULEN, R.; TANG, X.; LI, G.; et al. Comparison of hematological alterations and markers of B-cell activation in workers exposed to benzene, formaldehyde and trichloroethylene. Carcinogenesis, v. 37, n. 7, p. 692700, 2016. Disponível em: https://doi.org/10.1093/carcin/bgw053. Acesso em: 09 nov. 2021. doi: 10.1093/carcin/bgw053.

BRASIL - Ministério do Trabalho e Emprego. Norma Regulamentadora 15 Atividades e Operações Insalubres. Brasil: Portaria SEPRT $n . .1 .359$, de 09 de dezembro de 2019. Disponível em: https://www.gov.br/trabalho-e-previdencia/ptbr/composicao/orgaos-especificos/secretaria-de-trabalho/inspecao/seguranca-esaude-no-trabalho/normas-regulamentadoras/nr-15-anexo-03.pdf. Acesso em: 10 fev. 2021.

CETESB - Companhia Ambiental do Estado de São Paulo. Ficha de Informação Toxicológica. São Paulo: CETESB, 2017. Disponível em: https://cetesb.sp.gov.br/laboratorios/servicos/informacoes-toxicologicas/. Acesso em: 05 nov. 2021.

COGLIANO, V. J.; GROSSE, Y.; BAAN, R. A.; STRAIF, K.; SECRETAN, M. B.; et al. Meeting report: summary of IARC monographs on formaldehyde, 2-butoxyethanol, and 1-tert-butoxy-2-propanol. Environmental Health Perspectives, v. 113, n. 9, p. 1205-1208, 2005. Disponível em: https://doi.org/10.1289/ehp.7542. Acesso em: 09 nov. 2021.

DFG - Deutsche Forschungsgemeinschaft. Toxikologisch-arbeitsmedizinische Begründungen von MAK-Werten. Weinheim: Wiley VCH-Verlag, 2000. Disponível em:

https://www.dfg.de/download/pdf/dfg_magazin/gremien_politikberatung/gesundheitss chutz/50_jahre_mak.pdf. Acesso em: 15 set. 2020. 
DIODIU, R.; GALAON, T.; BUCUR, E.; STEFAN, D. S.; PASCU, L. F. Aldehydes, acetone, formic and acetic acids in indoor air of an office building. Revista de Chimie, v. 67, n. 12, p. 1-4, 2016. Disponível em: http://www.dspace.incdecoind.ro/bitstream/123456789/1081/1/DIDOIU\%20R\%2012 \%2016.pdf. Acesso em: 06 nov. 2021.

DUGHERI, S.; BONARI, A.; POMPILIO, I.; COLPO, M.; MUCCI, N.; ARCANGELI, G. An integrated air monitoring approach for assessment of formaldehyde in the workplace. Safety and Health at Work, v. 9, n. 4, p. 479-485, 2018. Disponível em: https://doi.org/10.1016/j.shaw.2018.05.002. Acesso em: 06 nov. 2021. doi: 10.1016/j.shaw.2018.05.002.

ESCALONA, L.; MANGANIELLO, L.; LÓPEZ-FONSECA, M.; VEGA, C. Los sensores químicos y su utilidad en el control de gases contaminantes. Revista Ingenueía UC, v. 19, n. 1, p. 74-88, 2012. Disponível em: https://www.redalyc.org/articulo.oa?id=70732261010. Acesso em: 06 nov. 2021.

FENG, L.; LIU, Y.; ZHOU, X.; HU, J. The fabrication and characterization of a formaldehyde odor sensor using molecularly imprinted polymers. Journal of Colloid and Interface Science, v. 284, n. 2, p. 378-382, 2005. Disponível em:https://doi.org/10.1016/j.jcis.2004.10.054. Acesso em: 06 nov. 2021. doi: 10.1016/j.jcis.2004.10.054.

FERREIRA, A. M. C.; CARDOSO, S. M. Estudo exploratório da qualidade do ar em escolas de educação básica, Coimbra, Portugal. Revista de Saúde Pública, v. 47, p. 1059-1068, 2013. Disponível em: https://doi.org/10.1590/S00348910.2013047004810. Acesso em: 06 nov. 2021. doi: 10.1590/S00348910.2013047004810 .

FU, X.; YANG, O.; XIAO, X.; ZHOU, D.; HUANG, R.; et al. Ultra-fast and highly selective room-temperature formaldehyde gas sensing of Pt-decorated $\mathrm{MoO} 3$ nanobelts. Journal of Alloys and Compounds, v. 797, p. 666-675, 2019. Disponível em: https://doi.org/10.1016/j.jallcom.2019.05.145. Acesso em: 06 nov. 2021. doi: 10.1016/j.jallcom.2019.05.145.

GODISH, T. Residential formaldehyde sampling-current and recommended practices. American Industrial Hygiene Association Journal, v. 46, n. 3, p. 105110, 2010. Disponível em: https://doi.org/10.1080/15298668591394509. Acesso em: 06 nov. 2021. doi: 10.1080/15298668591394509.

HALKET, J. M.; ZAIKIN, V. G. Derivatization in mass spectrometry-5. Specific derivatization of monofunctional compounds. European Journal of Mass Spectrometry, v. 11, n. 1, p. 127-160, 2005. Disponível em: https://doi.org/10.1255/ejms.712. Acesso em: 06 nov. 2021. doi: 10.1255/ejms.712.

HLADOVÁ, M.; MARTINKA, J.; RANTUCH, P.; NECAS, A. Review of spectrophotometric methods for determination of formaldehyde. Journal of Materials Science \& Technology, v. 27, p. 105-120, 2019. Disponível em: https://www.researchpapers.mtf.stuba.sk/wp-

content/uploads/2019/11/VP44_12_2019_Hladov\%C3\%A1.pdf. Acesso em: 06 nov. 
2021. doi: 10.2478/rput-2019-0012.

JSOH - Japan Society for Occupational Health. Recommendation of Occupational Exposure Limits. Japão: Journal of Occupational Health, n. 40 p. 178-193, 1998. Disponível em: https://www.sanei.or.jp/?mode=view\&cid=310. Acesso em: 10 fev. 2021.

KABIR, E.; KIM, K.-H. An investigation on hazardous and odorous pollutant emission during cooking activities. Journal of Hazardous Materials, v. 188, n. 1-3, p. 443454, 2011. Disponível em: https://doi.org/10.1016/j.jhazmat.2011.01.113. Acesso em: 06 nov. 2021. doi: 10.1016/j.jhazmat.2011.01.113.

KLEPEIS, N. E.; NELSON, W. C.; OTT, W. R.; ROBINSON, J. P.; TSANG, A. M.; et al.The National Human Activity Pattern Survey (NHAPS): a resource for assessing exposure to environmental pollutants. Journal of Exposure Science \& Environmental Epidemiology, v. 11, n. 3, p. 231-252, 2001. Disponível em: https://doi.org/10.1038/sj.jea.7500165. Acesso em: 06 nov. 2021. doi: 10.1038/sj.jea.7500165.

LAGHRIB, F.; LAHRICH, S.; EL MHAMMEDI, M. A. Recent Advances in Direct and Indirect Methods for Sensing Carbonyl Compounds Aldehydes in Environment and Foodstuffs. Journal of the Electrochemical Society, v. 166, n. 15, p. B1543, 2019. Disponível em: https://doi.org/10.1149/2.0761915jes. Acesso em: 06 nov. 2021. doi: 10.1149/2.0761915jes.

LI, Y.; CAKMAK, S.; ZHU, J. Profiles and monthly variations of selected volatile organic compounds in indoor air in Canadian homes: Results of Canadian national indoor air survey 2012-2013. Environment International, v. 126, p. 134-144, 2019. Disponível em: https://doi.org/10.1016/j.envint.2019.02.035. Acesso em: 06 nov. 2021. doi: 10.1016/j.envint.2019.02.035.

LOWINSOHN, D.; BERTOTTI, M. Sensores eletroquímicos: considerações sobre mecanismos de funcionamento e aplicações no monitoramento de espécies químicas em ambientes microscópicos. Química Nova, v. 29, p. 1318-1325, 2006. Disponível em: https://doi.org/10.1590/S0100-40422006000600029. Acesso em: 06 nov. 2021. doi: 10.1590/S0100-40422006000600029.

MAJCHRZAK, T.; WOJNOWSKI, W.; LUBINSKA-SZCZYGEL, M.; RÓZANSKA, A.; NAMISESNIK, J.; DYMERSKI, T. PTR-MS and GC-MS as complementary techniques for analysis of volatiles: A tutorial review. Analytica Chimica Acta, v. 1035, p. 1-13, 2018. Disponível em: https://doi.org/10.1016/j.aca.2018.06.056. Acesso em: 06 nov. 2021. doi: 10.1016/j.aca.2018.06.056.

MARCHAND, C.; BULLIOT, B.; LE CALVÉ, S.; MIRABEL, P. Aldehyde measurements in indoor environments in Strasbourg (France). Atmospheric Environment, v. 40, n. 7, p. 1336-1345, 2006. Disponível em: https://doi.org/10.1016/j.atmosenv.2005.10.027. Acesso em: 06 nov. 2021. doi: 10.1016/j.atmosenv.2005.10.027.

MENDELL, M. J.; MIRER, A.; LEI-GOMEZ, Q. Technical Report: Contaminants in 
Buildings and Occupied Spaces as Risk Factors for Occupant Symptoms in U.S. Office Buildings: Findings from the U.S. EPABASE Study. Laboratório Nacional de Lawrence $\quad$ Berkeley. 63370, $2007 . \quad$ Disponível em: https://www.osti.gov/servlets/purl/928793. Acesso em: 07 nov. 2021.

MIKSCH, R.; ANTHON, D. W.; FANNING, L. Z.; HOLLOWELL, C. D.; REVZAN, K.; et al. Modified pararosaniline method for the determination of formaldehyde in air. Analytical Chemistry, v. 53, n. 13, p. 2118-2123, 1981. Disponível em: https://doi.org/10.1021/ac00236a040. Acesso em: 08 nov. 2021. doi: 10.1021/ac00236a040.

MOEL - Ministry of Employment and Labor. Exposure criteria to chemicals and physical agents, 2010. Disponível em: https://www.moel.go.kr/english/pas/pasMOEL.jsp\#. Acesso em: 15 out. 2020.

MOKALLED, T.; GÉRARD, J. A.; ABBOUD, M.; TROCQUET, C.; NASREDDINE, R.; et al. VOC tracers from aircraft activities at Beirut Rafic Hariri International Airport. Atmospheric Pollution Research, v. 10, n. 2, p. 537-551, 2019. Disponível em: https://doi.org/10.1016/j.apr.2018.09.009. Acesso em: 09 nov. 2021. doi: 10.1016/j.apr.2018.09.009.

NIOSH - National Institute of Occupational and Safety Health. NIOSH Pocket Guide to Chemical Hazards. Washington DC: US Gov Printing Office, 1994. Disponível em: https://www.cdc.gov/niosh/npg/default.html. Acesso em: 15 out. 2020.

NUNES, C. R. de O.; SÁNCHEZ, B.; GATTS, C. E. N.; DE ALMEIDA, C. M. S.; CANELA, M. C. Evaluation of volatile organic compounds coupled to seasonality effects in indoor air from a commercial office in Madrid (Spain) applying chemometric techniques. Science of the Total Environment, v. 650, p. 868-877, 2019. Disponível em: https://doi.org/10.1016/j.scitotenv.2018.09.095. Acesso em: 05 nov. 2021. doi: 10.1016/j.scitotenv.2018.09.095.

OBERMEIER, J.; TREFZ, P.; WEX, K.; SABEL, B.; SCHUBERT, J. K.; MIEKISCH, W. Electrochemical sensor system for breath analysis of aldehydes, $\mathrm{CO}$ and $\mathrm{NO}$. Journal of Breath Research, v. 9, n. 1, p. 016008, 2015. Disponível em: https://doi.org/10.1088/1752-7155/9/1/016008. Acesso em: 06 nov. 2021. doi: 10.1088/1752-7155/9/1/016008.

OLMO, N. R. S.; PEREIRA, L. A. A. Poluição atmosférica e exposição humana: A epidemiologia influenciando as políticas públicas. InterfacEHS - Revista de Saúde, Meio Ambiente e Sustentabilidade, v. 6, n. 2, p. 2-8. Disponível em: http://www3.sp.senac.br/hotsites/blogs/InterfacEHS/wpcontent/uploads/2013/08/2_ARTIGO_vol6n2.pdf. Acesso em: 10 out. 2021.

OSHA - Occupational Safety and Health Administration. Occupational Exposure to Hazardous Chemicals in Laboratories: Laboratory Standard 29 CRF 1910.1450. USA: OSHA, 1990. Disponível em: https://www.osha.gov/lawsregs/regulations/standardnumber/1910/1910.1450. Acesso em: 15 out. 2020.

PASETTO, L. V.; SIMON, V.; RICHARD, R.; PIC, J. S.; VIOLLEAU, F.; MANERO, M. 
$\mathrm{H}$. Aldehydes gas ozonation monitoring: Interest of SIFT/MS versus GC/FID. Chemosphere, v. 235, p. 1107-1115, 2019. Disponível em: https://doi.org/10.1016/j.chemosphere.2019.06.186. Acesso em: 06 nov. 2021. doi: 10.1016/j.chemosphere.2019.06.186.

PERRAUD, V.; MEINARDI, S.; BLAKE, D. R.; FINLAYSON-PITTS, B. J. Challenges associated with the sampling and analysis of organosulfur compounds in air using real-time PTR-ToF-MS and offline GC-FID. Atmospheric Measurement Techniques, v. 9, n. 3, p. 1325-1340, 2016. Disponível em: https://doi.org/10.5194/amt-9-1325-2016. Acesso em: 06 nov. 2021. doi: 10.5194/amt-9-1325-2016.

REINGRUBER, H.; PONTEL, L. B. Formaldehyde metabolism and its impact on human health. Current Opinion in Toxicology, v. 9, p. 28-34, 2018. Disponível em: https://doi.org/10.1016/j.cotox.2018.07.001. Acesso em: 06 nov. 2021. doi: 10.1016/j.cotox.2018.07.001.

ROTHWEILER, H.; WÄGER, P. A.; SCHLATTER, C. Comparison of Tenax TA and Carbotrap for sampling and analysis of volatile organic compounds in air. Atmospheric Environment. Part B. Urban Atmosphere, v. 25, n. 2, p. 231-235, 1991. Disponível em: https://doi.org/10.1016/0957-1272(91)90058-M. Acesso em: 06 nov. 2021. doi: 10.1016/0957-1272(91)90058-M.

SAKAMOTO, T.; DOI, S.; TORII, S. Effects of formaldehyde, as an indoor air pollutant, on the airway. Allergology International, v. 48, n. 3, p. 151-160, 1999. Disponível em: https://doi.org/10.1046/j.1440-1592.1999.00131.x. Acesso em: 06 nov. 2021. doi: 10.1046/j.1440-1592.1999.00131.x.

SALTHAMMER, T.; MENTESE, S.; MARUTZKY, R. Formaldehyde in the indoor environment. Chemical Reviews, v. 110, n. 4, p. 2536-2572, 2010. Disponível em: https://doi.org/10.1021/cr800399g. Acesso em: 06 nov. 2021. doi: $10.1021 / \mathrm{cr} 800399 \mathrm{~g}$.

SÁNCHEZ, B.; SÁNCHEZ-MUÑOZ, M.; MUÑOZ-VICENTE, M.; COBAS, G.; PORTELA, R.; et al. Photocatalytic elimination of indoor air biological and chemical pollution in realistic conditions. Chemosphere, v. 87, n. 6, p. 625-630, 2012. Disponível em: https://doi.org/10.1016/j.chemosphere.2012.01.050. Acesso em: 06 nov. 2021. doi: 10.1016/j.chemosphere.2012.01.050.

SÁNCHEZ, B.; SOUZA, M. de O.; VILANOVA, O.; CANELA, M. C. Volatile organic compounds in the Spanish National Archaeological Museum: Four years of chemometric analysis. Building and Environment, v. 174, p. 106780, 2020. Disponível em: https://doi.org/10.1016/j.buildenv.2020.106780. Acesso em: 06 nov. 2021. doi: 10.1016/j.buildenv.2020.106780.

SCHIEWECK, A.; GUNSCHERA, J.; VAROL, D.; SALTHAMMER, T. Analytical procedure for the determination of very volatile organic compounds (C 3-C 6) in indoor air. Analytical and Bioanalytical Chemistry, v. 410, n. 13, p. 3171-3183, 2018. Disponível em: https://doi.org/10.1007/s00216-018-1004-z. Acesso em: 06 nov. 2021. doi: 10.1007/s00216-018-1004-z. 
SCHIRMER, W. N.; PIAN, L. B.; SZYMANSKI, M. S. E.; GAUER, M. A. A poluição do ar em ambientes internos e a síndrome dos edifícios doentes. Ciência \& Saúde Coletiva, v. 16, p. 3583-3590, 2011. Disponível em: https://doi.org/10.1590/S141381232011000900026. Acesso em: 06 nov. 2021. doi: 10.1590/S141381232011000900026 .

SOFUOGLU, S. C.; ASLAN, G.; INAL, F.; SOFUOGLU, A. An assessment of indoor air concentrations and health risks of volatile organic compounds in three primary schools. International Journal of Hygiene and Environmental Health, v. 214, n. 1, p. 36-46, 2011. Disponível em: https://doi.org/10.1016/j.ijheh.2010.08.008. Acesso em: 06 nov. 2021. doi: 10.1016/j.jheh.2010.08.008.

SOUSA, F. C.; TINÔCO, I. F. F.; SARAZ, J. A. O.; SOUZA, C. F.; PAULA, M. O.; et al. Métodos de Difusão Passiva para Quantificação de Amônia em Instalações Abertas para Produção Animal/Methods of Passive Diffusion for Ammonia Determination in open Facilities for Animal Production. Revista Brasileira de Engenharia de Biossistemas (Online), v. 9, n. 3, p. 252-260, 2015. Disponível: https://doi.org/10.18011/bioeng2015v9n3p252-260. Acesso em: 07 nov. 2021. doi: 10.18011/bioeng2015v9n3p252-260.

SOUZA, M. de O.; VIEIRA, H. G.; SÁNCHEZ, B.; CANELA, M. C. O Estado da Arte de Compostos Carbonílicos Voláteis em Ambientes Internos: Impactos à Saúde e Metodologias de Amostragem e Análises. Química Nova, v. 44, p. 830-844, 2021. Disponível em: https://doi.org/10.21577/0100-4042.20170741. Acesso em: 07 nov. 2021. doi: 10.21577/0100-4042.20170741.

SOUZA, M. de O.; SÁNCHEZ, B.; FUENTES, M.; GILARANZ, J.; CANELA, M. C. Analytical validation using a gas mixing system for the determination of gaseous formaldehyde. Analytical Methods, v. 12, n. 43, p. 5247-5256, 2020. Disponível em: https://doi.org/10.1039/D0AY01363C. Acesso em: 07 nov. 221. doi: 10.1039/D0AY01363C.

TRINKLEY, M. Considerações sobre preservação na construção e reforma de bibliotecas: planejamento para preservação. Rio de Janeiro: CPBA: Arquivo Nacional, ed. 2, 2001. Disponível em: https://egov.df.gov.br/wpcontent/uploads/2019/09/Cadernos-t\%C3\%A9cnicos-CPBA-\%E2\%80\%93-38-

\%E2\%80\%93-Preserva\%C3\%A7\%C3\%A3o-constru\%C3\%A7\%C3\%A3o-ereformas.pdf. Acesso em: 07 nov. 2021.

TSAI, W.-T. An overview of health hazards of volatile organic compounds regulated as indoor air pollutants. Reviews on Environmental Health, v. 34, n. 1, p. 81-89, 2019. Disponível em: https://doi.org/10.1515/reveh-2018-0046. Acesso em: 07 nov. 2021. doi: 10.1515/reveh-2018-0046.

USEPA - United States Environmental Protection Agency. Compendium of Methods for the Determination of Toxic Organic Compounds in Ambient Air. USA: $\quad$ EPA/625/R- 96/010b, 53, 1999. Disponível em: https://www3.epa.gov/ttnamti1/files/ambient/airtox/to-15r.pdf. Acesso em: 15 out. 2020. 
VERONEZ, D. A. da L.; FARIAS, E. L. P.; DE FRAGA, R.; DE FREITAS, R. S.; PETERSEN, M. L.; SILVEIRA, J. R. de P. Potencial de Risco para a Saúde Ocupacional de Docentes, Pesquisadores e Técnicos de Anatomia Expostos ao Formaldeído. InterfacEHS, v. 5, n. 2, 2010. Disponível em: http://www3.sp.senac.br/hotsites/blogs/InterfacEHS/wpcontent/uploads/2013/08/4_ARTIGO_vol5n2.pdf. Acesso em: 05 nov. 2021.

VOGEL, M.; BÜLDT, A.; KARST, U. Hydrazine reagents as derivatizing agents in environmental analysis-a critical review. Fresenius' Journal of Analytical Chemistry, v. 366, n. 8, p. 781-791, 2000. Disponível em: https://doi.org/10.1007/s002160051572. Acesso em: 07 nov. 2021. doi: $10.1007 / \mathrm{s} 002160051572$.

WHO - World Health Organization. Biological monitoring of chemical exposure in the workplace: guidelines. Geneva, 1996. Disponível em: https://apps.who.int/iris/handle/10665/41856. Acesso em: 08 ago. 2020.

WOLKOFF, P. Indoor air pollutants in office environments: assessment of comfort, health, and performance. International Journal of Hygiene and Environmental Health, v. 216, n. 4, p. 371-394, 2013. Disponível em: https://doi.org/10.1016/j.ijheh.2012.08.001. Acesso em: 07 nov. 2021. doi: 10.1016/j.ijheh.2012.08.001.

YON, Y.; RAMIRO-GONZALEZ, M.; MIKTON, C. R.; HUBER, M.; SETHI, D. The prevalence of elder abuse in institutional settings: a systematic review and metaanalysis. European Journal of Public Health, v. 29, n. 1, p. 58-67, 2019. Disponível em: https://doi.org/10.1093/eurpub/cky093. Acesso em: 08 nov. 2021. doi: 10.1093/eurpub/cky093.

ZHANG, S.; SHAPIRO, N.; GEHRKE, G.; CASTNER, J.; LIU, Z.; GUO, B.; et al. Smartphone app for residential testing of formaldehyde (SmART-form). Building and Environment, v. 148, p. 567-578, 2019. Disponível em: https://doi.org/10.1016/j.buildenv.2018.11.029. Acesso em: 07 nov. 2021. doi: 10.1016/j.buildenv.2018.11.029. 